\title{
Metamaterial-Inspired Rotation Sensor With Wide Dynamic Range
}

\author{
Amir Ebrahimi, Student Member, IEEE, Withawat Withayachumnankul, Said F. Al-Sarawi, Member, IEEE, \\ and Derek Abbott, Fellow, IEEE
}

\begin{abstract}
A rotation sensor with a wide dynamic range is designed based on tapered $U$-shaped resonators. The proposed device is composed of a rounded microstrip transmission line that couples to two meandered resonators that are stacked on top of each other. By rotating the upper resonator, the overlapping area between the two resonators is increased causing a stronger coupling that shifts down the resonance frequency of the device. This frequency shift can be read out in the transmission response from which the rotation angle is determined. The operation principle of the sensor is explained in detail by using a circuit model. A sensor prototype is designed for the microwave frequency range and an experiment is presented for validating the proposed sensing approach. This sensing device is well suited for further miniaturization using microelectromechanical systems technology.
\end{abstract}

Index Terms-Metamaterial-inspired sensor, rotation sensor, split-ring resonator.

\section{INTRODUCTION}

$\mathbf{M}$ ETAMATERIALS are artificially engineered materials made of sub-wavelength resonators that can manipulate the electromagnetic waves to cause some exotic electromagnetic properties [1]. Since their introduction, they have been applied to many new or improved electromagnetic designs such as microwave filters [2], [3], left handed transmission lines [4] and antenna designs [5], [6]. A resonator as a metamaterial unit cell is typically made of a metallic open loop ring with a capacitive gap to provide a high quality resonance. The resonance of these resonators is very sensitive to surrounding materials and dimensional changes [7], [8]. These properties make them attractive choices for sensing applications. Various types of metamaterial-based sensors have been introduced so far for different applications from microwave to terahertz frequency ranges such as: microfluidic sensors [9], [10], microwave biological sensors [11], [12], strain sensors [13], [14], thin film sensors [15]-[19].

Recently, new alignment and rotation sensors have been introduced [20], [21] based on split-ring resonator (SRR) coupled coplanar waveguide (CPW). In these configurations, displacing or rotating the SRR from the CPW symmetry

Manuscript received December 20, 2013; revised February 14, 2014; accepted March 19, 2014. Date of publication March 25, 2014; date of current version June 26, 2014. The associate editor coordinating the review of this paper and approving it for publication was Dr. Kailash Thakur.

The authors are with the School of Electrical and Electronic Engineering, University of Adelaide, Adelaide, SA 5005, Australia (e-mail: amir.ebrahimi@adelaide.edu.au).

Color versions of one or more of the figures in this paper are available online at http://ieeexplore.ieee.org.

Digital Object Identifier 10.1109/JSEN.2014.2313625 plane causes a resonance in the transmission response of the structure, where the resonance notch depth is function of either the displacement or rotation of the SRR. Further, a tapered diamond shaped SRR in [22] and a horn shaped $\mathrm{SRR}$ in [23] are proposed with a fixed resonance frequency. However, in all of the previous rotation sensors, the dynamic range is limited to around 6-8 degrees. A rotation sensor based on an electric-LC (ELC) resonator coupled CPW has been introduced bay Naqui and Martin [24] that increases the dynamic range up to 90 degrees. In this paper, a new concept of metamaterial-inspired rotation sensors is introduced with improvement in the dynamic range up to 180 degrees. Advantages of the proposed design in comparison with conventional rotation sensors (e.g. rotary encoders and potentiometers) include a wider dynamic range, lower fabrication cost, and MEMS compatibility. The sensor is designed based on two meandered U-shaped resonators coupled to a microstrip line. The rotation changes the coupling coefficient between the two U-shaped resonators causing a resonance frequency shift in the transmission response of the structure from which the rotation angle can be read out. In addition to the dynamic range improvement, the sensor linearity is also improved by asymmetrically tapering the resonator shapes. The design principle and experiment are presented in sections II and III, respectively.

\section{SENSOR DESIGN PRINCIPLE}

\section{A. Preliminary Design}

A top and a cross sectional views of the proposed rotation sensor are given in Fig. 1. As shown, the sensor is composed of two identical U-shaped resonators that are coupled to a single microstrip line. The first resonator is positioned on the same substrate with the microstrip line, whereas the second U-shaped resonator is positioned on another substrate located on the top of the first substrate, as shown in the cross-sectional view in Fig 1(b). The middle part of the microstrip line is rounded to provide an optimum coupling area for the resonators. The two substrates are separated from each other by a very thin air gap. The upper substrate can freely rotate relative to the bottom one. If the upper substrate is rotated around the axis as shown in Fig. 1(a), the overlapping area between the resonators will be increased resulting in stronger electric and magnetic coupling between the two resonators. Effectively, the resonance frequency will be shifted, from which the rotation angle can be determined. 


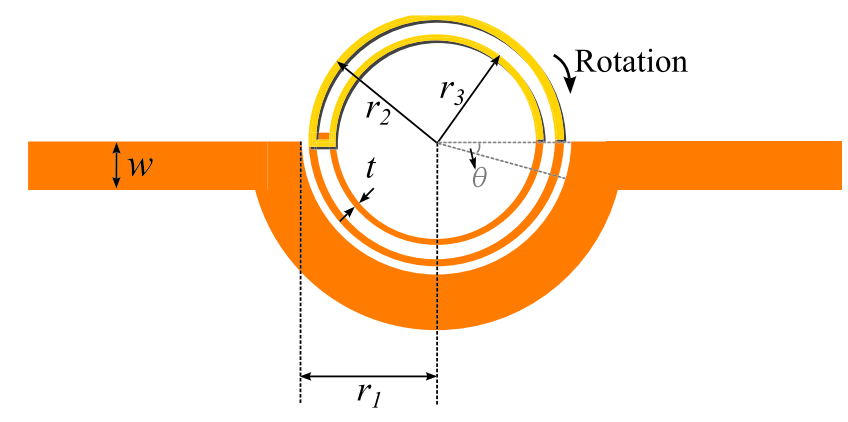

(a)

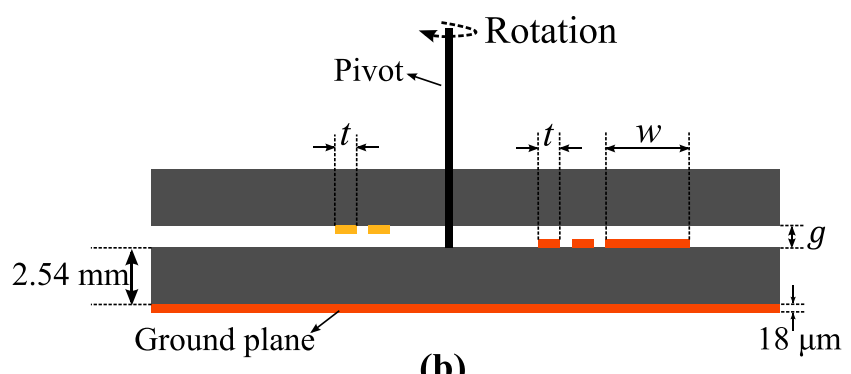

(b)

Fig. 1. Schematic of the rotation sensor. (a) Top view with removed dielectric substrates for clarity. (b) Cross section of the proposed sensor. The yellow part indicates the upper resonator metallization, and the orange shows the lower resonator and the microstrip line metallization. The top and bottom substrates are indicated with gray color. The dimensions are: $w=8.3 \mathrm{~mm}, t=0.3 \mathrm{~mm}$, $r_{1}=15 \mathrm{~mm}, r_{2}=14.8 \mathrm{~mm}, r_{3}=12.6 \mathrm{~mm}$ and $g=0.25 \mathrm{~mm}$.

This phenomenon can be explained by using the circuit model of the structure.

The equivalent circuit model of the two coupled U-shaped resonators is presented in Fig. 2(a), where $L_{r}$ represents the equivalent inductance of the resonators and $C_{r}$ corresponds to the equivalent capacitance. Since the dimensions of the two U-shaped resonators are exactly the same, the equivalent circuit parameters are the same for them. As shown, $C_{M}$ is the mutual capacitance and $L_{M}$ is the mutual inductance between the two resonators. The $L_{M}$ and $C_{M}$ values are proportinal to the overlapping area and inversely proportional to the distance between the two resonators. By using a simple circuit analysis, the circuit in Fig. 2(a) can be simplified to circuit shown in Fig. 2(b), where the capacitive coupling is modelled by a $\pi$ equivalent model and inductive coupling is replaced by its equivalent $T$ circuit model [25]. By further simplification, the circuits of Fig. 2(c) and (d) are obtained that show the even and odd-mode resonances respectively. Based on Fig. 2(c) and (d), the even and odd-mode resonance frequencies can be found to be

$$
\begin{aligned}
& f_{\text {even }}=\frac{1}{2 \pi \sqrt{L_{e} C_{e}}}=\frac{1}{2 \pi \sqrt{\left(L_{r}-L_{M}\right)\left(C_{r}-C_{M}\right)}}, \\
& f_{\text {odd }}=\frac{1}{2 \pi \sqrt{L_{o} C_{o}}}=\frac{1}{2 \pi \sqrt{\left(L_{r}+L_{M}\right)\left(C_{r}+C_{M}\right)}} .
\end{aligned}
$$

By increasing the overlapping area between the two resonators, the values of $L_{M}$ and $C_{M}$ are increased. As a result, the even-mode resonance frequency will move upward, whereas the odd-mode resonance frequency will be shifted

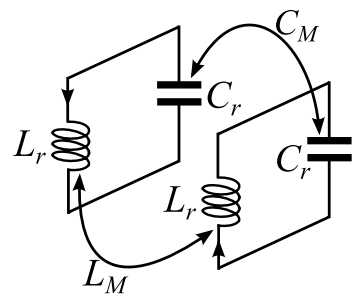

(a)

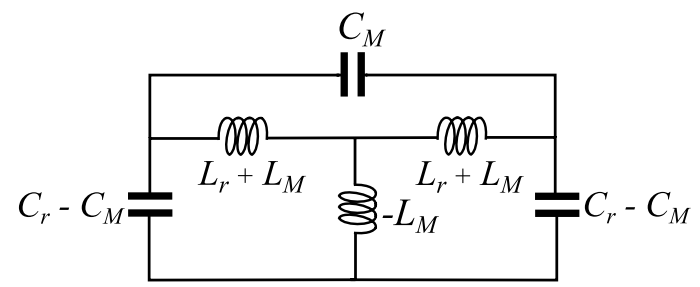

(b)

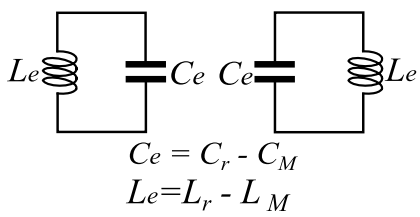

(c)

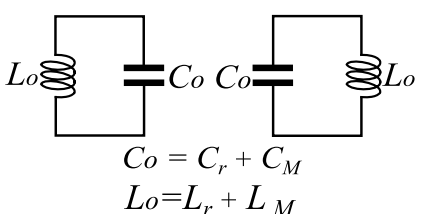

(d)
Fig. 2. Equivalent circuit models. (a) Circuit model of the two coupled U-shaped meandered resonators. (b) Simplified model of the two coupled U-shaped resonators. (c) Equivalent circuit for even-mode resonance. (d) Equivalent circuit for odd-mode resonance.

downwards. Here, the odd-mode resonance change is exploited to determine the rotation angle since it yields a more compact sensor with respect to the operating wavelength. This resonance frequency change can be observed in the transmission amplitude $\left(\left|S_{21}\right|\right)$ of of the transmission line. An equivalent circuit for the resonator-loaded microstrip line is shown in Fig. 3(a) where, $L$ and $C$ are the parameters associated with the microstrip, and $M$ is the coupling coefficient between the microstrip line and the resonator [26]. In order to validate the circuit of Fig. 3(a), the equivalent circuit parameters have been calculated for a sample rotation angle of 80 degrees by using the method presented in [27]. The obtained parameters are fed into the ADS circuit simulator and the result is compared with that obtained from Momentum ADS electromagnetic simulation. The dielectric substrate is Rogers 5880LZ with a low relative permittivity of $\epsilon_{r}=1.96$ to increase the freespace electric fringing field and hence improving the capacitive coupling between the two resonators. The loss tangent of the substrate is 0.0019. As shown in Fig. 3(b), the results are in a good agreement confirming the validity of the proposed circuit model.

As a proof of the sensing concept, the sensor is fully simulated in Momentum ADS by using the dimensions and parameters given in Fig. 1. The electromagnetic simulations results of the sensor are shown in Fig. 4. As seen, the sensor is able to sense a rotation angle up to 180 degrees. The limitation of this design is the nonlinearity of the device response. As demonstrated, the resonance frequency shift is relatively 


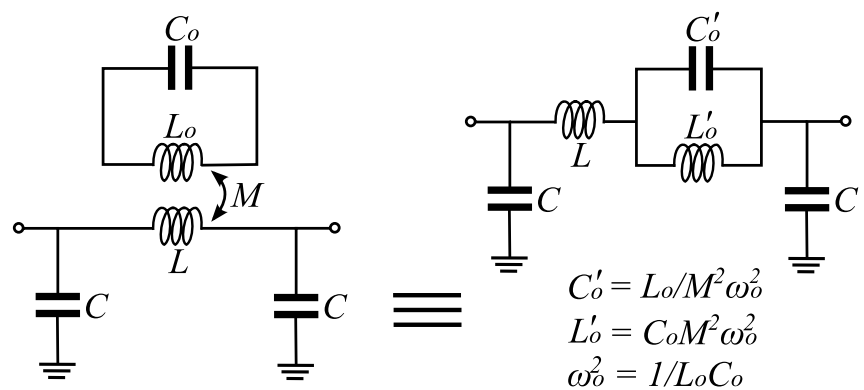

(a)

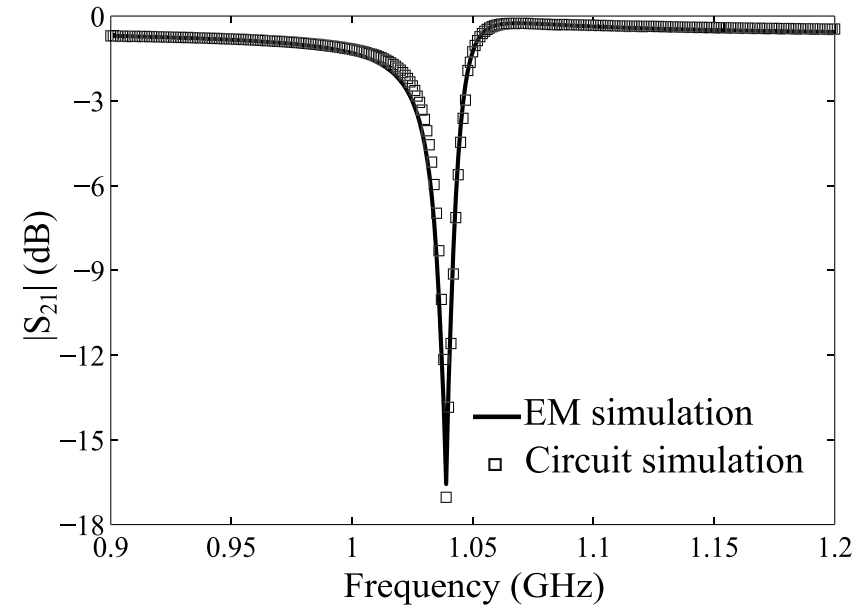

(b)

Fig. 3. Operation of the sensor. (a) Equivalent circuit model of the proposed rotation sensor in the odd-mode resonance. (b) Comparison between the equivalent circuit and electromagnetic simulation results for the rotation angle of 80 degree. The circuit parameters are: $L_{o}^{\prime}=0.0974 \mathrm{nH}, C_{o}^{\prime}=240.6 \mathrm{pF}$, $L=8.85 \mathrm{nH}$ and $C=2.13 \mathrm{pF}$. The quality factor of the resonance is 200 if it is defined as $f_{0} / \Delta f$ where $\Delta f$ is the bandwidth at $+3 \mathrm{~dB}$ with respect to the minimal transmission.

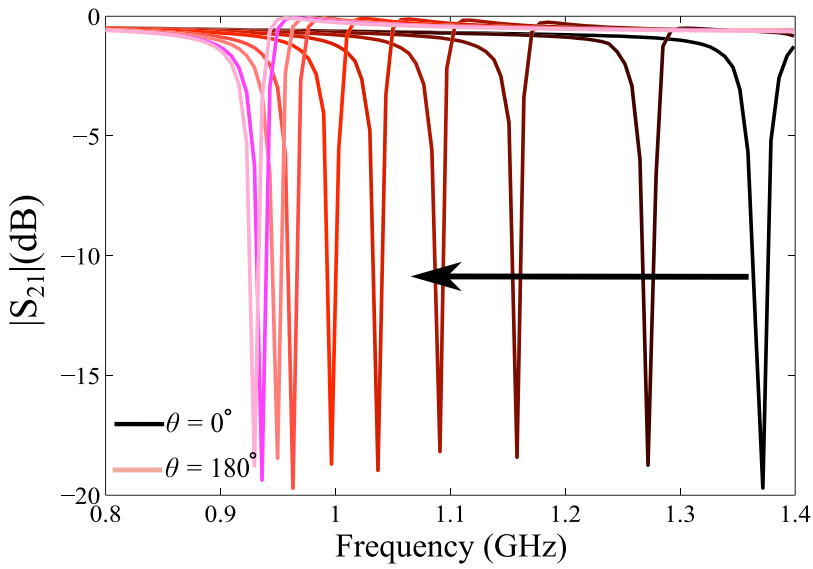

Fig. 4. Simulation results of the transmission coefficient for the designed sensor of Fig. 1 for different rotation angles up to 180 degrees. The rotation step size is 20 degrees.

large between 0 and 80 degrees, implying high sensitivity. However, the frequency shift and hence the sensitivity reduce significantly beyond 80 degrees. This nonlinearity is due to the uniform shape of the two resonators. In fact, with this uniform shape the overlapping area between the two resonators varies

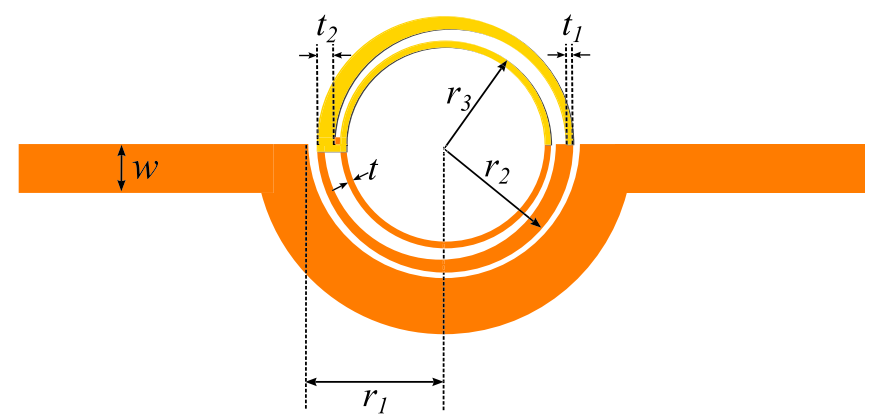

Fig. 5. The enhanced design for improving the linearity of sensor response. The dimensions are: $w=8.3 \mathrm{~mm}, t=t_{1}=0.3 \mathrm{~mm}, r_{1}=15 \mathrm{~mm}$, $r_{2}=14.8 \mathrm{~mm}, r_{3}=12.6 \mathrm{~mm}$ and $t_{2}=1.7 \mathrm{~mm}$.

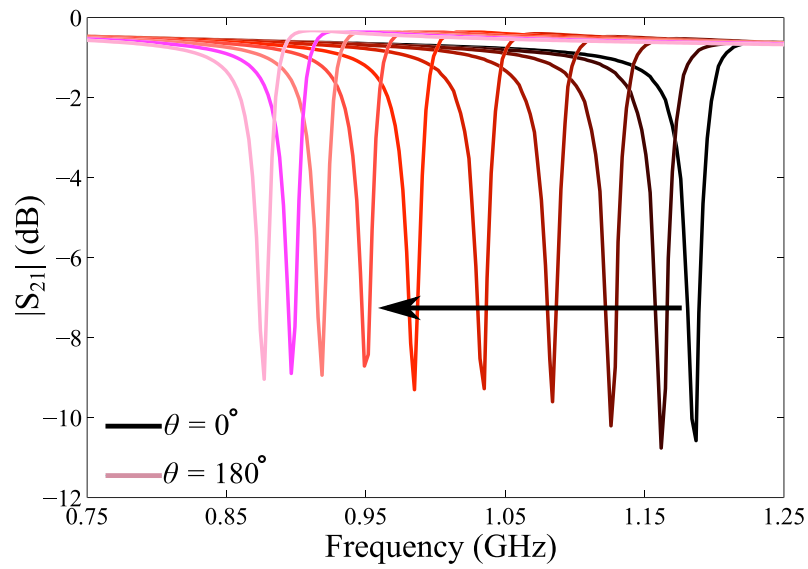

Fig. 6. Simulated transmission coefficient for the improved sensor in Fig. 5 for different rotation angles up to 180 degrees. The rotation step size is 20 degrees.

linearly when the upper resonator is rotated with respect to the bottom one and this causes a linear change in $L_{o} C_{o}$. But, the linear change in the $L_{o} C_{o}$ causes a nonlinear shift in resonance frequency as suggested by the relation in (2).

\section{B. Linearizing the Sensor Response}

In order to improve the sensor linearity, the overlapping area of the two resonators should be changed nonlinearly as a function of the rotation angle. This can be carried out by modifying the resonators shape. An improved design is illustrated in Fig. 5. In this structure, the outer arms of the two U-shaped resonators are asymmetrically tapered. Using this approach, the overlapping area of the two resonators will be developed slowly at the beginning of rotation. As a result, the mutual inductance and capacitance changes will be small. By increasing the rotation angle, tapering causes a sharper change in the overlapping area hence, the mutual inductance and capacitance changes will be larger and the sensitivity will be increased for the large rotation angles around 180 degrees.

Fig. 6 depicts the simulated resonance frequency shifts for the improved design of Fig. 5 at different rotation angles. As seen, the modified sensor shows better sensitivity for large rotation angles close to 180 degrees. For a better comparison in terms of linearity, the resonance frequencies for the sensor 


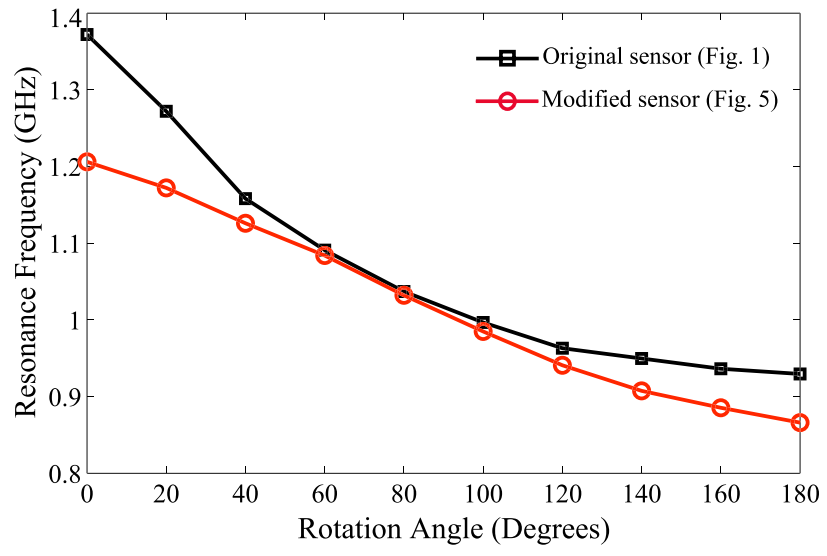

Fig. 7. Comparison between the simulated resonance frequency of the simple design in Fig. 1 and the improved design in Fig. 5 for different rotation angles.

in Fig. 1 and the modified structure in Fig. 5 are plotted versus the rotation angle in Fig. 7. As shown, the structure in Fig. 5 offers an improved linearity in comparison with the initial design in Fig. 1.

\section{Sensitivity Analysis}

The sensitivity of the rotation sensor can be defined as

$$
S_{\theta}^{f_{0}}=\frac{\theta}{f_{0}} \frac{d f_{0}}{d \theta}
$$

where, $S_{\theta}^{f_{0}}$ is the sensitivity of the rotation sensor with respect to the rotation angle, $f_{0}$ is the resonance frequency of the sensor and $\theta$ is the rotation angle. From (3) and Fig. 7, the sensitivity of the simple design in Fig. 1 at three rotation angles of 20, 90 and 180 degrees are calculated as $1.1,0.18$ and 0.03 respectively. On the other hand, the sensitivity of the improved sensor in Fig. 5 at these three rotation angles are calculated as $0.98,0.93$ and 0.89 respectively. The above analysis clearly demonstrates that the improved design in Fig. 5 retains a constant sensitivity of around 0.9 over 0 to 180 degrees rotation angle, while the sensitivity of the simple sensor of Fig. 1 is high at small rotation angles and very small at large rotation angles around 180 degrees showing the sensitivity saturation. It should be mentioned that the improved linearity of the modified design in Fig. 5 is achieved at the expense of reduced sensitivity for small rotation angles.

\section{Detecting the Rotating Direction}

The proposed sensor can be used to sense the rotation direction. For this application, the zero (starting) point of the rotation can be set to 90 degrees as shown in Fig. 8. In this arrangement, if the upper resonator is rotated clockwise, the overlapping area of the two resonators will be increased causing a stronger coupling between the two resonators, hence the resonance frequency shifts downwards. On the other hand, if the upper resonator is rotated counterclockwise, the coupling between the two resonators will be decreased and the resonance frequency moves upwards. In this way, the rotation angles within \pm 90 degrees can be detected.

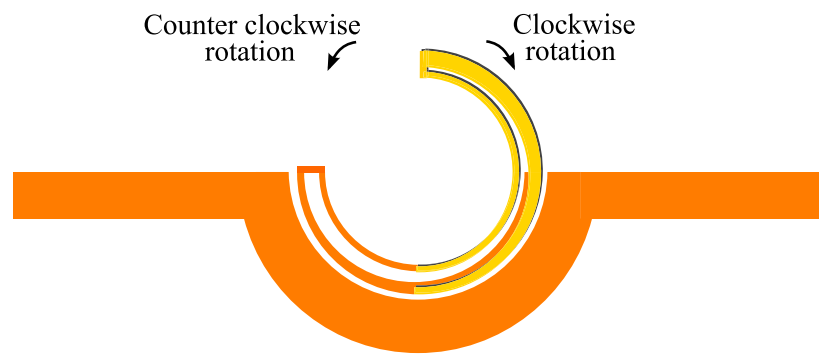

Fig. 8. Sensor arrangement for detecting the direction of rotation.

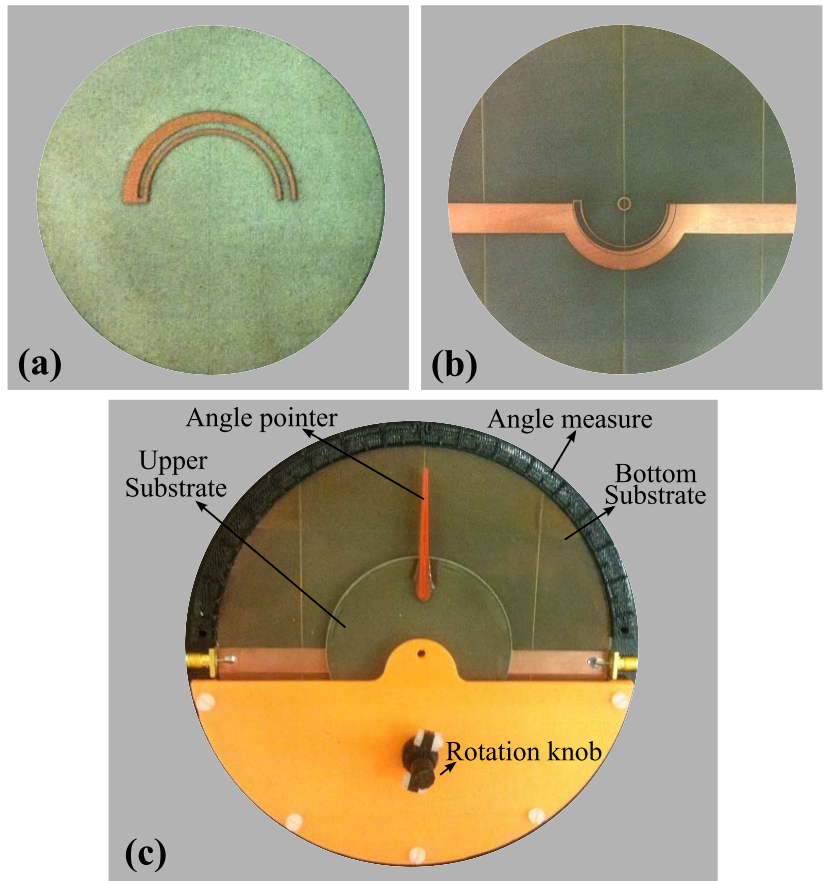

Fig. 9. The fabricated sensor module. (a) The upper plate containing a resonator. (b) The lower plate containing a resonator coupled with a microstrip line. (c) The assembled sensor module, packaged with parts obtained from $3 \mathrm{D}$ printing. A rotation knob connects to the pivot through set of gears for rotating the upper resonator with respect to the bottom one. The angle scale and pointer are included for setting the rotation angle accurately.

\section{EXPERIMENTAL RESULTS}

In order to verify the rotation sensing concept presented in the previous section and validate the simulation results of the improved sensor design, a sensor prototype has been fabricated based on the dimensions given in Fig. 5. The device is fabricated on Rogers 5880LZ dielectric substrate. Fig. 9 illustrates the fabricated sensor.

Fig. 10 shows the measured transmission coefficient $\left(S_{21}\right)$ of the fabricated sensor for different rotation angles. In each measurement step, the upper plate is rotated using the rotating knob and the transmission response is recorded by using the network analyzer. In contrast to the simple configuration in Fig. 1, the improved sensor design shows a linear frequency shift for large rotation angles close to 180 degrees while maintaining a compact size with respect to the operational wavelength. Fig. 11 compares the measured and simulated resonance frequencies as a function of the rotation angle. The plot 


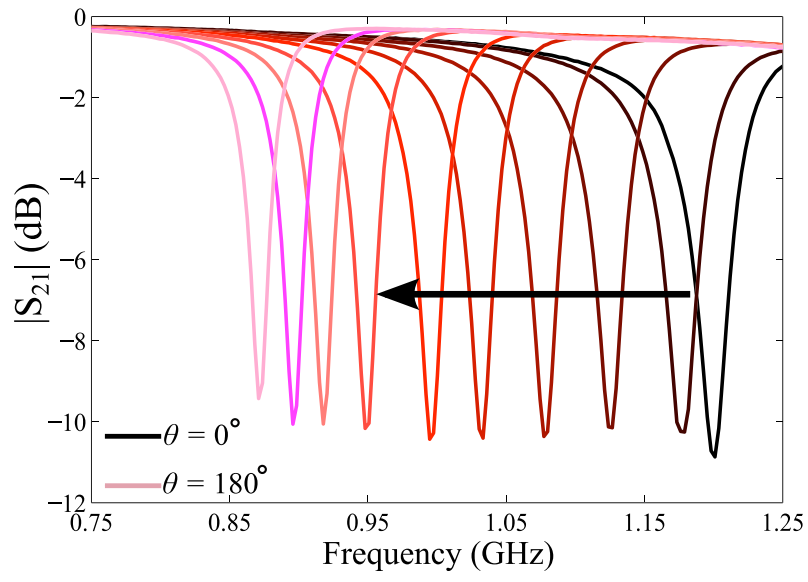

Fig. 10. Measurments results of the transmission coeficient for the fabricated sensor prototype fo different rotation angles up to 180 degrees. The rotation angle step size is 20 degrees.

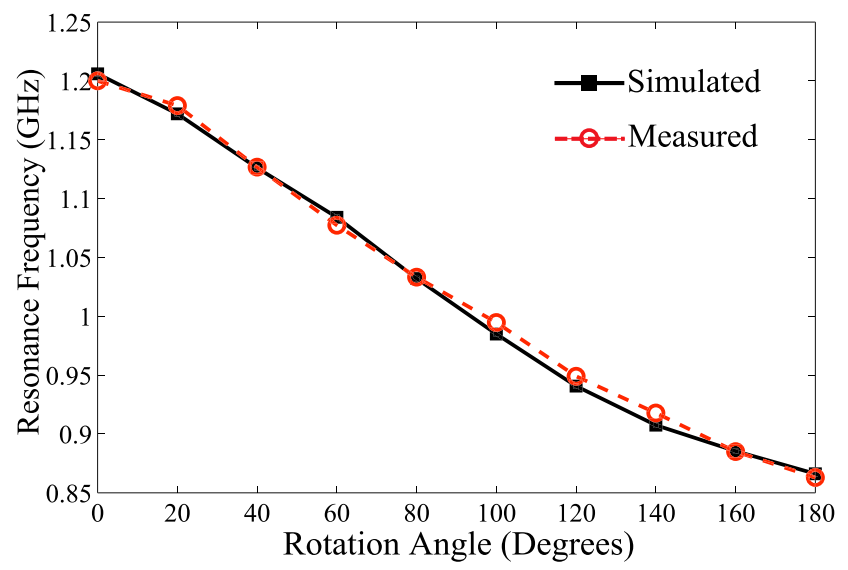

Fig. 11. Comparison between the measured and simulated resonance frequency of the improved designed sensor in Fig. 5.

shows a good agreement between the simulated and measured results. The differences between the measured and simulated responses can be attributed to the fabrication and assembling tolerances. Based on these results, the proposed rotation sensing concept and the improved design for linearizing the sensor response are validated.

\section{CONCLUSION}

A wide dynamic range rotation sensor based on broad side coupled U-shaped resonators has been proposed and analyzed by using an equivalent circuit model. To improve the sensitivity and linearity of the sensor response, a modified design based on asymmetrically tapered resonators is developed and verified by simulations and experimental measurement. The results show the sensor ability to detect rotation angles up to 180 degrees.

\section{ACKNOWLEDGMENT}

The authors would like to thank P. Simcik and I. Linke for their technical assistance in fabricating and assembling the sensor.

\section{REFERENCES}

[1] T. Chen, S. Li, and H. Sun, "Metamaterials application in sensing," Sensors, vol. 12, no. 3, pp. 2742-2765, 2012.

[2] J. Bonache, I. Gil, J. García-García, and F. Martín, "Novel microstrip bandpass filters based on complementary split-ring resonators," IEEE Trans. Microw. Theory Tech., vol. 54, no. 1, pp. 265-271, Jan. 2006.

[3] A. Ebrahimi, W. Withayachumnankul, S. Al-Sarawi, and D. Abbott, "Compact dual-mode wideband filter based on complementary splitring resonator," IEEE Microw. Wireless Compon. Lett., vol. 24, no. 3, pp. 152-154, Mar. 2014.

[4] M. Gil, J. Bonache, J. García-García, J. Martel, and F. Martín, "Composite right/left-handed metamaterial transmission lines based on complementary split-rings resonators and their applications to very wideband and compact filter design," IEEE Trans. Microw. Theory Tech., vol. 55, no. 6, pp. 1296-1304, Jun. 2007.

[5] E. Pucci, E. Rajo-Iglesias, M. Kehn, and O. Quevedo-Teruel, "Enhancing the efficiency of compact patch antennas composed of split-ring resonators by using lumped capacitors," IEEE Antennas Wireless Propag. Lett., vol. 11, pp. 1362-1365, 2012.

[6] N. Takemura, "Tunable inverted-L antenna with split-ring resonator structure for mobile phones," IEEE Trans. Antennas Propag., vol. 61, no. 4, pp. 1891-1897, Apr. 2013.

[7] N. I. Zheludev, "The road ahead for metamaterials," Science, vol. 328 , no. 5978, pp. 582-583, 2010.

[8] S.-Y. Chiam, R. Singh, W. Zhang, and A. A. Bettiol, "Controlling metamaterial resonances via dielectric and aspect ratio effects," Appl. Phys. Lett., vol. 97, no. 19, p. 191906, 2010.

[9] W. Withayachumnankul, K. Jaruwongrungsee, A. Tuantranont, C. Fumeaux, and D. Abbott, "Metamaterial-based microfluidic sensor for dielectric characterization," Sensors Actuat. A, Phys., vol. 189, pp. 233-237, Jan. 2013.

[10] A. Ebrahimi, W. Withayachumnankul, S. Al-Sarawi, and D. Abbott, "High-sensitivity metamaterial-inspired sensor for microfluidic dielectric characterization," IEEE Sensors J., vol. 14, no. 5, pp. 1345-1351, May 2014.

[11] M. Puentes, M. Maasch, M. Schussler, and R. Jakoby, "Frequency multiplexed 2-dimensional sensor array based on split-ring resonators for organic tissue analysis," IEEE Trans. Microw. Theory Tech., vol. 60, no. 6, pp. 1720-1727, Jun. 2012.

[12] H.-J. Lee et al., "A planar split-ring resonator-based microwave biosensor for label-free detection of biomolecules," Sensors Actuat. B, Chem., vol. 169, pp. 26-31, Jul. 2012.

[13] R. Melik, E. Unal, N. K. Perkgoz, C. Puttlitz, and H. V. Demir, "Metamaterial-based wireless strain sensors," Appl. Phys. Lett., vol. 95, no. 1, pp. 011106-1-011106-3, Jul. 2009.

[14] J. Li et al., "Flexible terahertz metamaterials for dual-axis strain sensing," Opt. Lett., vol. 38, no. 12, pp. 2104-2106, 2013.

[15] I. A. I. Al-Naib, C. Jansen, and M. Koch, "Thin-film sensing with planar asymmetric metamaterial resonators," Appl. Phys. Lett., vol. 93, no. 8, p. $083507,2008$.

[16] W. Withayachumnankul, K. Jaruwongrungsee, C. Fumeaux, and D. Abbott, "Metamaterial-inspired multichannel thin-film sensor," IEEE Sensors J., vol. 12, no. 5, pp. 1455-1458, May 2012.

[17] W. Withayachumnankul et al., "Sub-diffraction thin-film sensing with planar terahertz metamaterials," Opt. Exp., vol. 20, no. 3, pp. 3345-3352, 2011.

[18] J. F. O'Hara et al., "Thin-film sensing with planar terahertz metamaterials: Sensitivity and limitations," Opt. Exp., vol. 16, no. 3, pp. 1786-1795, 2008.

[19] T. Driscoll et al., "Tuned permeability in terahertz split-ring resonators for devices and sensors," Appl. Phys. Lett., vol. 91, no. 6, p. 062511, 2007.

[20] J. Naqui, M. Duránn-Sindreu, and F. Martín, "Novel sensors based on the symmetry properties of split ring resonators (SRRs)," Sensors, vol. 11, no. 8, pp. 7545-7553, 2011.

[21] J. Naqui, M. Durán-Sindreu, and F. Martín, "Alignment and position sensors based on split ring resonators," Sensors, vol. 12, no. 9, pp. 11790-11797, 2012.

[22] A. Horestani, C. Fumeaux, S. Al-Sarawi, and D. Abbott, "Displacement sensor based on diamond-shaped tapered split ring resonator," IEEE Sensors J., vol. 13, no. 4, pp. 1153-1160, Apr. 2013.

[23] A. Horestani, D. Abbott, and C. Fumeaux, "Rotation sensor based on horn-shaped split ring resonator," IEEE Sensors J., vol. 13, no. 8, pp. 3014-3015, Aug. 2013. 
[24] J. Naqui and F. Martín, "Transmission lines loaded with bisymmetric resonators and their application to angular displacement and velocity sensors," IEEE Trans. Microw. Theory Tech., vol. 61, no. 12, pp. 4700-4713, Dec. 2013.

[25] J.-S. G. Hong and M. J. Lancaster, Microstrip Filters for RF/Microwave Applications, vol. 167. New York, NY, USA: Wiley, 2004.

[26] J. Baena et al., "Equivalent-circuit models for split-ring resonators and complementary split-ring resonators coupled to planar transmission lines," IEEE Trans. Microw. Theory Tech., vol. 53, no. 4, pp. 1451-1461, Apr. 2005.

[27] F. Aznar et al., "Characterization of miniaturized metamaterial resonators coupled to planar transmission lines through parameter extraction," J. Appl. Phys., vol. 104, no. 11, pp. 114501-1-114501-8, Dec. 2008.

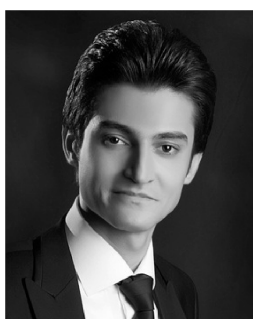

Amir Ebrahimi (S'09) was born in Babol, Iran, in 1986. He received the B.Sc. degree in electrical engineering from the University of Mazandaran, Babol, Iran, in 2008, and the M.Sc. degree in electronic engineering from the Babol University of Technology, Babol, Iran, where he was a Research Assistant with the Integrated Circuits Research Laboratory, from 2009 to 2012. He is currently pursuing the $\mathrm{Ph} . \mathrm{D}$. degree in electrical and electronic engineering at the University of Adelaide, Australia. His research interests include metamaterial-inspired microwave devices, microwave circuit design, microwave filters, and frequency selective surfaces. Mr. Ebrahimi is a recipient of an International Postgraduate Research (Ph.D.) Scholarship, Australian Postgraduate Award, and the Australian National Fabrication Facility Award for fabricating high-performance microwave microfluidic sensors in collaboration with the Ian Wark Research Institute, University of South Australia.

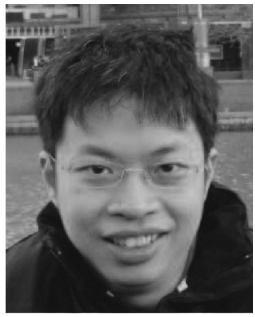

Withawat Withayachumnankul received the B.Eng. and M.Eng. degrees in electronic engineering from the King Mongkuts Institute of Technology Ladkrabang (KMITL), Bangkok, Thailand, in 2001 and 2003, respectively, and the Ph.D. degree in electrical engineering (with commendation) from the University of Adelaide, Adelaide, Australia, in 2010. From 2003 to 2012, he served as a Lecturer with the Faculty of Engineering, KMITL. Since 2010, he has held an ARC Australian Post-Doctoral Fellowship with the University of Adelaide. He is an Associate with RMIT University, Melbourne, Australia. His research interests include terahertz technology, metamaterials, plasmonics, and optical antennas.

Dr. Withayachumnankul has authored and co-authored more than 30 journal publications. He has delivered invited talks at ETH Zurich, University of Marburg, EPFL, IPHT, Germany, University of Western Australia, and Ibaraki University. He serves as a Grant Assessor with the Swiss National Science Foundation and Australian Research Council. He is a recipient of the 2008 IEEE/LEOS Graduate Student Fellowship, the 2008 SPIE Scholarship in Optical Science and Engineering, the Australian Endeavour International Postgraduate Research Scholarship from 2006 to 2008, and the SPIE Poster Award in 2007.

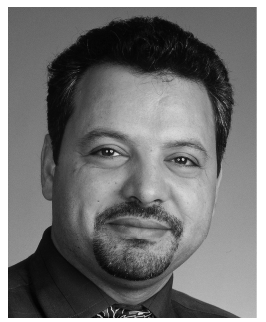

Said F. Al-Sarawi (M'92) received the General Certificate in marine radio communication and the B.Eng. (Hons.) degree in marine electronics and communication from the Arab Academy for Science and Technology, Egypt, and the Ph.D. degree in mixed analog and digital circuit design techniques for smart wireless systems with special commendation in electrical and electronic engineering from the University of Adelaide, Australia, in 1987, 1990, and 2003 , respectively. He was awarded the University of Adelaide Alumni Postgraduate Medal (formerly Culross Prize) for outstanding academic merit at the post-graduate level. While pursuing the $\mathrm{Ph} . \mathrm{D}$. degree, he received the Commonwealth Post-Graduate Research Award (Industry) and the Graduate Certificate in Education (Higher Education), in 2006, from the University of Adelaide. Currently, he is the Director of the Centre for Biomedical Engineering and a Founding Member of the Education Research Group of Adelaide. His research interests include design techniques for mixed signal systems in CMOS and optoelectronic technologies for high-performance radio transceivers, low-power and lowvoltage radio frequency identification systems, data converters, mixed signal design, and microelectromechanical systems for biomedical applications. His current educational research is focused on innovative teaching techniques for engineering education, research skill development, and factors affecting students evaluations of courses in different disciplines.

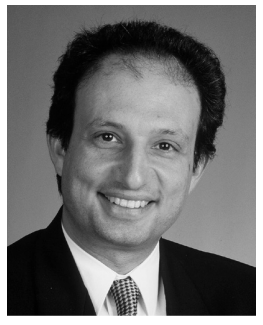

Derek Abbott (M'85-SM'99-F'05) was born in South Kensington, London, U.K., in 1960. He received the B.Sc. (Hons.) degree in physics from Loughborough University, U.K., and the Ph.D. degree in electrical and electronic engineering from the University of Adelaide, Australia, in 1982 and 1995, respectively, under the supervision of $\mathrm{K}$. Eshraghian and B. R. Davis. From 1978 to 1986, he was a Research Engineer with the GEC Hirst Research Centre, London, U.K. From 1986 to 1987, he was a VLSI Design Engineer with Austek Microsystems, Australia. Since 1987, he has been with the University of Adelaide, where he is currently a Full Professor with the School of Electrical and Electronic Engineering. He holds more than 800 publications/patents and has been an Invited Speaker at more than 100 institutions. He is a Fellow of the Institute of Physics. He was a recipient of a number of awards, including the South Australian Tall Poppy Award for Science in 2004, the Premier's SA Great Award in Science and Technology for outstanding contributions to South Australia in 2004, and an Australian Research Council Future Fellowship in 2012. He has served as an Editor and/or Guest Editor for a number of journals, including the IEEE JOURNAL OF SOLID-STATE CIRCUITS, the IEEE Journal of Optics B, the IEEE Microelectronics Journal, Chaos, Smart Structures and Materials, the IEEE Journal OF Optics B, and the IEEE Fluctuation Noise LetTers, and is currently on the editorial boards of the PROCEEDINGS OF THE IEEE, the IEEE PHOTONICS JOURNAL, and PLOS ONE. He co-edited Quantum Aspects of Life (Imperial College Press), and co-authored Stochastic Resonance (Cambridge University Press) and Terahertz Imaging for Biomedical Applications (Springer-Verlag). Prof. Abbott's interests are in the area of multidisciplinary physics and electronic engineering applied to complex systems. His research programs span a number of areas of stochastics, game theory, photonics, biomedical engineering, and computational neuroscience. 